\title{
Advanced FIB Sample Preparation for High Performance TEM Analysis
}

\author{
F. Altmann, R. Salzer, M. Simon, S. Huebner, Ch. Grosse, A. Graff
}

Fraunhofer Institute for Mechanics of Materials, Walter-Huelse-Strasse 1, 06120 Halle, Germany

Transmission electron microscopy (TEM) is becoming more and more important for industrial quality control and failure analysis. Today focused ion beam (FIB) preparation is the most common technique to produce electron transparent TEM samples from a specific area of interest. Especially for industrial applications the preparation time has to be as short as possible to enhance analysis throughput and to save costs. On the other hand atomic resolved imaging and high sensitive element analysis by electron energy loss spectroscopy (EELS) require ultrathin and mostly artifact free lamellas. With these terms the FIB preparation of such lamellas becomes more challenging. Monitoring of the real lamella thickness during thinning gives the possibility to better control the FIB milling and helps to avoid the destruction of the lamella during the end polishing step.

In combined SEM/FIB systems the preparation progress can be imaged during FIB milling. If the energy of the primary electrons is high enough to penetrate through the lamella the secondary electron (SE) intensity increases while the amount of back scattered electrons (BSE) decreases. These transparency effects depend on material parameter, primary electron energy and lamella thickness. The advantage of the observation of the BSE intensity is that it is not affected by FIB generated electrons. An approach was developed to utilize the BSE contrast for a generalized and standard free thickness measurement of electron transparent lamellas [1]. The method is based on a global normalized transparency function (GNTF) that was derived from Monte-Carlo simulations $[2,3]$. The GNTF represents the correlation between the normalized BSE coefficient and the thickness depending on primary electron energy and material parameters. It is used to correlate the lamella thickness with the BSE image intensity. A software tool was developed to visualize the thickness distribution of a TEM lamella as a color coded map [1]. The software is directly adapted to a Crossbeam ESB system from Zeiss NTS. It can be used for mapping of the remaining lamella thickness and end pointing during FIB milling. Now the lamella can continuously polished until the required thickness is reached. This makes the FIB milling process faster and more controllable. Furthermore inhomogeneous thickness distributions at the lamella can be detected early and compensated. Additionally low energy ion polishing can be better adjusted to remove the defect layer at the lamella sidewalls leaving a certain thickness for TEM analysis.

Moreover a new clamp system was developed to transfer and fix TEM lamellas. The clamps consist of two parallel poly Si beams forming a 700nm slit to carry the lamella by only mechanically fixing. Several single and double clamp geometries have been designed to optimize adjustment and fixing of the lamella. The poly Si clamps are an inherent part of a specific designed poly Si structure which can be directly transferred into a TEM sample holder. The poly Si structure is fabricated by MEMS processing on a Silicon wafer and can be released by HF etching the sacrificial SiO2 layer. The stability of the $3 \mu \mathrm{m}$ thin poly $\mathrm{Si}$ structure is enhanced by a specific plating process to grow $\mathrm{Cu}$ or Gold from both sides onto the poly Si structure. To transfer the lamella out of the sample into the clamp we are using the Kleindiek lift out shuttle (LOS) equipped with a piezo driven gripper. In combination of both the transfer time of the TEM lamella to the TEM holder could be reduced to less than 5 minutes. Additionally gas assisted deposition or gluing of the TEM lamella can totally be avoided and the transport needle has not to be reshaped by FIB. 


\section{References}

[1] R. Salzer, A. Graff, F. Altmann, M. Simon Microsc. Microanal. 15 (Suppl. 2) (2009)

[2] D. Drouin et al. Scanning Vol. 29 Issue 3 (2007) 92-101

[3] N. W. M. Ritchie Surf. Interface Anal. Vol. 37 (2005) 1006-1011

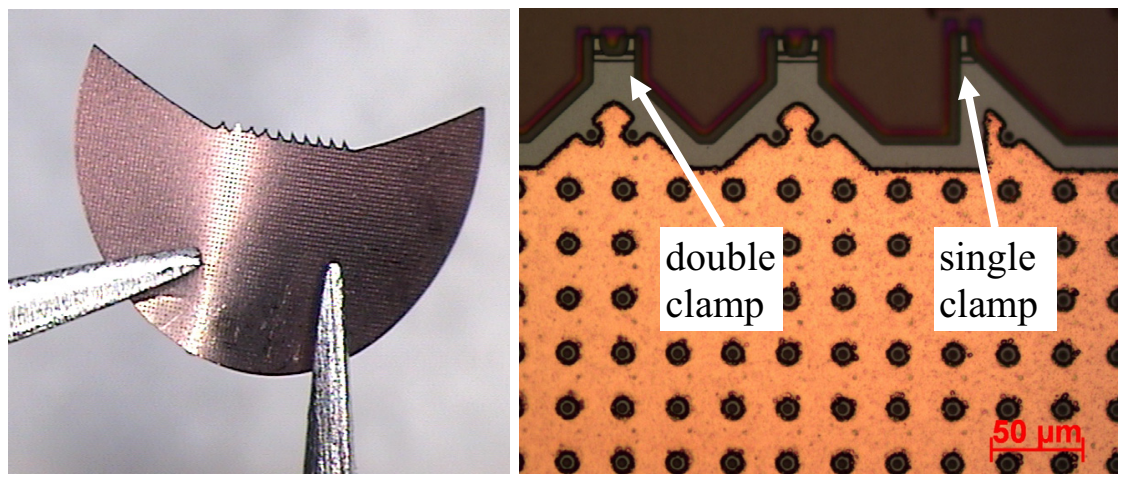

FIG. 1. Poly Si structure compatible to a TEM grid (left) with single and double clamps (right), $\mathrm{Cu}$ was grown onto poly Si to enhanced the mechanically stability

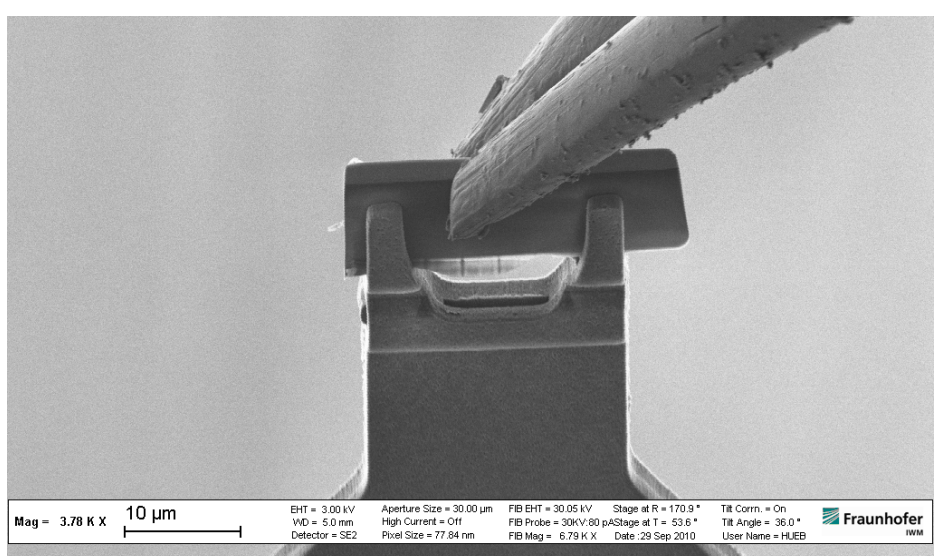

FIG. 2. TEM lamella is transferred into a double clamp using the piezo gripper attached to the Kleindiek lift out shuttle

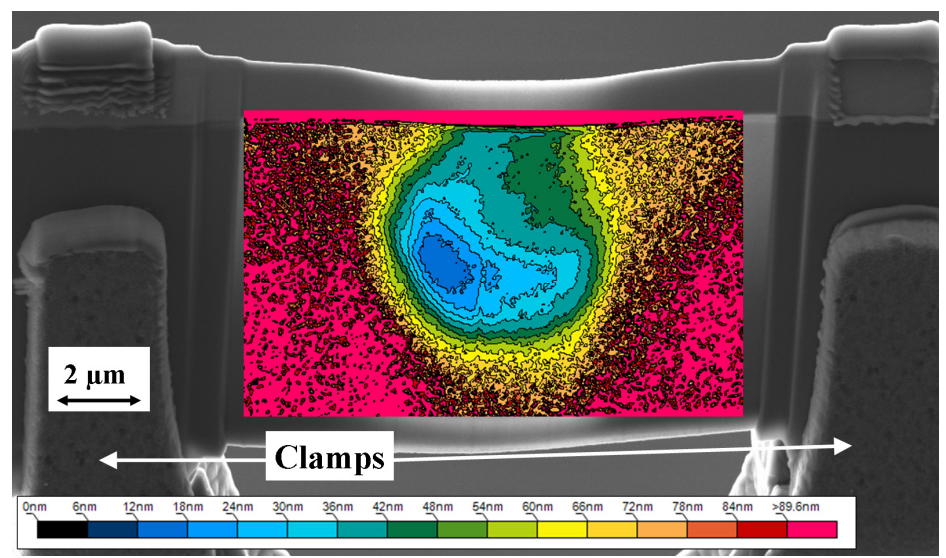

FIG. 3. SE image of a silicon lamella thinned by $2 \mathrm{kV}$ FIB to a residual thickness of about $10 \mathrm{~nm}$, the $\mathrm{SE}$ image is overlaid with a thickness map correlated from the corresponding BSE image at $4 \mathrm{keV}$. 